\title{
The Clinical Pharmacy Specialist: Part of the Solution
}

\author{
Adam J. Rose, MD MSc ${ }^{1,2}$, Megan B. McCullough, PhD ${ }^{3,4}$, Barry L. Carter, PharmD 5,6, and \\ Robert S. Rudin, $P h D^{7}$
}

'RAND Corporation, Boston, MA, USA; ${ }^{2}$ Department of Medicine, Section of General Internal Medicine, Boston University School of Medicine, Boston, MA, USA; ${ }^{3}$ Center for Healthcare Organization and Implementation Research, Bedford VA Medical Center, Bedford, MA, USA; ${ }^{4}$ Department of Health Policy and Management, Boston University School of Public Health, Boston, MA, USA; ${ }^{5}$ Department of Pharmacy Practice and Science, College of Pharmacy, University of lowa, lowa City, IA, USA; ${ }^{6}$ Department of Family Medicine, College of Medicine, University of lowa, lowa City, IA, USA.

$J$ Gen Intern Med 32(4):375-7

DOI: $10.1007 / \mathrm{s} 11606-016-3958-\mathrm{x}$

(c) Society of General Internal Medicine 2016

$\mathrm{P}$ rimary care is facing a shortage of providers, an issue compounded by expanded coverage under the Affordable Care Act (ACA) and population aging. Patients often cannot find a primary care panel that is accepting new patients, most practices cannot offer same-day appointments for acute complaints, and primary care providers (PCPs) may feel increasingly "burned-out". While the ACA contains provisions to expand the pool of PCPs over time, including improved compensation for primary care relative to other specialties, this sort of incentive would take years to work, and is unlikely to fully solve the problem. Team-based care is often advanced as a solution to improve the quality, accessibility, and sustainability of primary care. A recent article suggested that full implementation of team-based care could completely address primary care shortages, without adding new physicians, by delegating tasks to other team members. ${ }^{1}$ This article, like many others, focused on a healthcare team consisting of physicians, nurse practitioners, physician's assistants, and nurses. ${ }^{1}$ However, few articles about team-based primary care focus on the role of pharmacists, or even mention them-an omission also reflected in the setup of many practices.

This is a lost opportunity, because pharmacists can play a key role in "unloading" medication-related tasks from physicians. While no study has directly addressed exactly how much time PCPs spend managing medication therapy, one study found that family practitioners spent about one-third of chronic illness visits on "treatment planning". ${ }^{2}$ Much of this "treatment" is related to managing medication therapy; thus, relieving PCPs of this task could allow them to focus on other tasks for which their training is better suited.

Received September 8, 2016

Revised November 23, 2016

Accepted December 6, 2016

Published online December 19, 2016
A group of clinicians already exists who could assume some responsibility for managing medication therapy. Over the past 40 years, innovations in pharmacist education (the doctorate-level PharmD degree) and postdoctoral training (residency) have evolved in parallel with heightened expectations of what a pharmacist can and should be doing. The term "clinical pharmacy specialist (CPS)" is often used to describe pharmacists with enhanced training and skills. Nearly all CPSs have completed a PharmD degree as well as postdoctoral residency training. Many carry certifications such as Board Certified Pharmacotherapy Specialist (BCPS), analogous to certification by the American Board of Medical Specialties. In many settings, CPSs have legal authority to prescribe, order and follow laboratory tests, and generally perform all functions required to manage medication therapy.

The skills and training of a CPS can be most fully realized in the context of physician-pharmacist collaborative management. ${ }^{3}$ Physician-pharmacist collaborative management is more complex than routine medication therapy management. In physician-pharmacist collaborative management, the CPS may take responsibility for adjusting a medication (e.g., warfarin) in perpetuity, following a request from the PCP. Or the PCP can identify a patient with uncontrolled diabetes or hypertension, and ask the CPS to address the issue and send the patient back when the condition is under control. CPSs can help address other pressing problems, such as improving safety with opioids, managing medications for mental illness, and preventing readmissions in heart failure. CPSs are already fulfilling advanced roles in many settings, such as the Veterans Health Administration, large health maintenance organizations, and inpatient services. But there is an opportunity to expand these roles to additional settings - most prominently, as an important part of a truly high-functioning primary care team.

Studies show that pharmacists perform at least as well as physicians in managing chronic disease, measured by intermediate outcomes such as glycemic or blood pressure control. ${ }^{4}$ A key factor in their success may be their ability to see patients more frequently than a PCP. A relatively simple request (e.g., to bring hemoglobin A1c down from 13 to 8) can require ten or more distinct medication titrations to accomplish. It is important that these visits are carried out in a 
timely fashion. If providers cannot fit these visits in frequently enough, patients may spend longer periods of time with poorly controlled chronic conditions, increasing the risk of morbidity, mortality, and hospitalization. For example, if 12 visits are required to achieve a reduction in hemoglobin $\mathrm{A} 1 \mathrm{c}$ from 13 to 8 , weekly visits could accomplish the goal in just 3 months, while monthly visits would take a full year.

CPS roles are limited. For example, diagnosis is not the province of pharmacists. In a clinical setting, CPSs operate by referral from a physician. Initially, physicians may feel more comfortable originating these referrals themselves; however, it would also be possible to auto-generate a referral for any patient meeting certain criteria (e.g., hemoglobin A1c >9), or at least generate a referral for the physician to sign.

There are potential barriers to increasing the role of CPSs. Historically, lobbying by physician organizations has contributed to the enactment of laws in some states imposing restrictions on pharmacy practice. Beyond turf battles, physicians may have genuine concerns about whether pharmacists can take care of their patients, even though studies suggest that pharmacists can do it as well and sometimes better. ${ }^{4}$ These concerns tend to be most pronounced among physicians who work in non-academic settings or who have not previously collaborated with CPSs. However, physicians who do have experience working closely with a CPS often become strong advocates for the value of physician-pharmacist collaborative management. In any event, physicians will need time to become accustomed to working with a new team member and effectively coordinating care. Similarly, patients may be unfamiliar with this new member of the healthcare team, and may need orientation to their pharmacist's new responsibilities.

From a business perspective, a patchwork of state-level laws and regulations have led to variation in what pharmacists are allowed to do independently and, perhaps more importantly, the services for which pharmacists are able to bill. However, the advent of alternative payment models may encourage expanded pharmacist participation in primary care delivery, because these new payment systems shift the focus away from billing as a key determinant of what makes business sense. Even within a fee-for-service framework, there may be expanding opportunities for pharmacists to bill indirectly in states where they are granted limited authority. For example, the Centers for Medicare \& Medicaid Services now allow billing for chronic care management (CCM) or for medication reconciliation. A CPS can do most of this work, especially with a nurse for backup on non-medication items. One of us recently led a study of a centralized cardiovascular risk service, staffed by pharmacists, that provided remote services to primary care practices. ${ }^{5}$ This efficacy study has now been expanded into a free-standing (non-research) service, which continues to provide remote services to primary care. Payment for this program is now being pursued through billing for $\mathrm{CCM}$ and medication reconciliation codes. Private physicians have been highly supportive of this service, and have delegated a great deal of responsibility to the pharmacists. This experience contrasts with the commonly held belief that physicians in non-academic practices may resist the idea of delegating responsibility to a CPS.

Similarly, as physician practices become part of accountable care organizations or seek level 3 medical home recognition, more of their reimbursement will depend on achieving certain outcomes, such as controlling diabetes or preventing readmissions for heart failure. If pharmacists can achieve these outcomes faster, better, or at less cost, physician groups may see a strong business case for adding a CPS.

Several research efforts could advance our understanding of the potential benefits of expanding use of CPSs. First, states vary in the degree of autonomy they allow for CPSs. Studies should examine the impact of these state laws on the cost and quality of care. Second, research should examine how best to integrate CPSs into clinical workflows and teams, which could start by studying teams that already utilize CPSs particularly effectively. One important part of this effort may be to quantify what proportion of work in a primary care setting a CPS can feasibly do. Third, there is a need to evaluate what happens when a CPS is added to a practice that has not had one before, in terms of the impact on outcomes, workload, and the practice's bottom line. This would ideally be accomplished with a mixed-methods evaluation of a practice that is in the process of adding a CPS. Finally, while studies have quantified the impact of pharmacists on intermediate outcomes, ${ }^{5}$ it is unlikely that a study will be adequately powered to directly show an impact on definitive outcomes (e.g., myocardial infarction). Simulation models, linking pharmacist-accomplished improvements in intermediate outcomes to expected downstream benefits, could help strengthen the case for increasing the role of CPSs.

Clinical pharmacy specialists are important healthcare workers, but we may not be using their knowledge or skills to the fullest. CPSs can make valuable contributions to improving quality and access, while helping control costs. Efforts to create high-functioning primary care teams should recognize and take advantage of all that they have to offer.

Corresponding Author: Adam J. Rose, MD MSc; RAND Corporation, Boston, MA, USA (e-mail: arose@rand.org).

\section{Compliance with Ethical Standards:}

Funding: General support from the Bedford VA Medical Center to Dr. McCullough; no other funding.

Disclaimer: The opinions expressed in this article do not necessarily reflect the official policies of the US Department of Veterans Affairs.

Conflict of Interest: The authors declare that they have no conflict of interest.

\section{REFERENCES}

1. Auerbach DI, Chen PG, Friedberg MW, et al. Nurse-managed health centers and patient-centered medical homes could mitigate expected primary care physician shortage. Health Aff. 2013;11:1933-41. 
2. Yawn B, Goodwin MA, Zyzanski SJ, Stange KC. Time use during acute and chronic illness visits to a family physician. Fam Pract. 2003;20:474-7.

3. Carter BL. Primary care physician-pharmacist collaborative care model: strategies for implementation. Pharmacotherapy. 2016;36:363-73.
4. Greer N, Bolduc J, Geukink E, et al. Pharmacist-led chronic disease management: a systematic review of effectiveness and harms compared with usual care. Ann Intern Med. 2016;165:30-40.

5. Carter BL, Coffey CS, Ardery G, et al. Cluster-randomized trial of a physician/pharmacist collaborative model to improve blood pressure control. Circ Cardiovasc Qual Outcomes. 2015;8:235-243. 